\title{
Erythroderma and Chronic Lichenification Due to Metformin
}

\author{
Javier Moreno Díaz ${ }^{1}$, Jose Miguel García Bruñén ${ }^{1}$, Rocío Bermúdez Cameo², Ana Martínez González ${ }^{3}$ \\ ${ }^{1}$ Internal Medicine Department, University Hospital Miguel Servet, Zaragoza, Spain \\ ${ }^{2}$ Pathological Anatomy Department, University Hospital Miguel Servet, Zaragoza, Spain \\ ${ }^{3}$ Rehabilitation Department, Hospital of Barbastro, Huesca, Spain
}

Received: $14 / 04 / 2019$

Accepted: 28/04/2019

Published: 29/05/2019

\begin{abstract}
How to cite this article: Moreno Díaz J, García Bruñén JM, Bermúdez Cameo R, Martínez González A. Erythroderma and chronic lichenification due to
\end{abstract} metformin. EJCRIM 2019;6: doi:10.12890/2019_001119.

Conflicts of Interests: The Authors declare that there are no competing interests.

This article is licensed under a Commons Attribution Non-Commercial 4.0 License

\section{ABSTRACT}

We present the case of a 62-year-old man with a 4-year history of pruritus and erythroderma. The patient had been taking metformin for 5 years, so after contact allergies, contact with toxic products, and autoimmune diseases were ruled out, the condition was treated as erythroderma secondary to metformin. A skin biopsy subsequently confirmed the diagnosis and lichenification of some areas of the patient's skin.

\section{LEARNING POINTS}

- Erythroderma induced by drugs, even very common drugs, should be ruled out in all cases.

- Other causes of erythroderma should be investigated with exhaustive clinical history taking, specific tests and skin biopsy.

- Erythroderma should be treated as soon as possible once the cause is known.

\section{KEYWORDS}

Metformin, erythroderma, lichenification, pruritus

\section{INTRODUCTION}

Erythroderma or 'red skin', which is often associated with exfoliative dermatitis, is a skin condition that manifests as diffuse erythema with skin peeling (more than $90 \%$ of the skin is usually involved). It is associated with a wide variety of diseases with skin involvement such as psoriasis, atopic dermatitis, hypersensitivity reactions to drugs and cutaneous T-cell lymphomas (Sézary syndrome). Chronic forms can become lichenified, causing permanent skin alterations and requiring careful clinical management.

\section{CASE REPORT}

We describe the case of a 62-year-old man with history of type 2 diabetes mellitus who had been treated for 5 years with metformin 850 mg twice a day. He was a smoker (10 cigarettes/day) and did not consume alcohol regularly. He lived in an urban environment, worked in a furniture assembly company, and did have contact with toxic products or with animals.

The patient was referred to the internal medicine clinic for evaluation of his skin lesions which presented as erythema, increased skin peeling and intense itching. The condition was progressive, had developed over about 4 years and currently affected more than $90 \%$ of his skin, with worsening symptoms despite topical treatments based on corticosteroids and antihistamines.

On examination, the skin showed cutaneous erythema together with an increase in the reticular pattern, as well as desquamation of the palms of the hands but without involvement of the nails (Figs. 1 and 2). Screening tests for autoimmune diseases and other diseases such as porphyrias, and serotypes for hepatotropic viruses and HIV were all negative. 

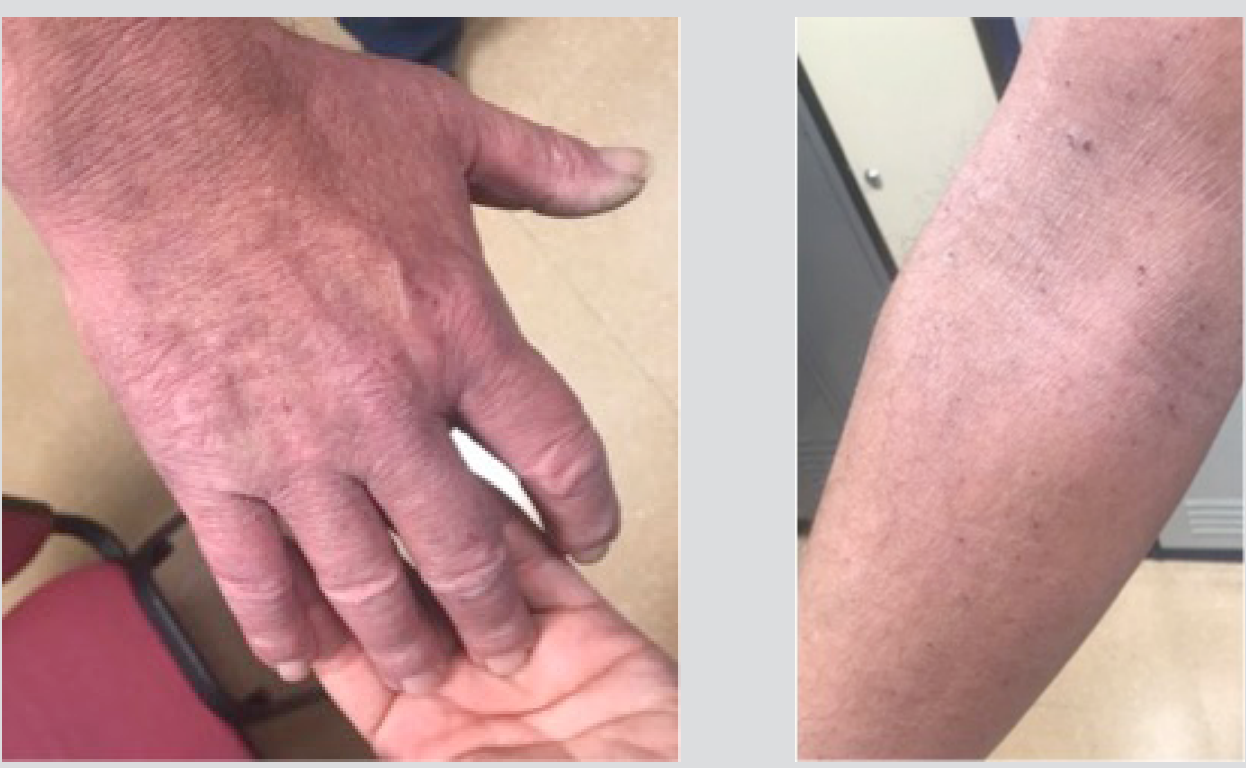

Figure 1. Hardened erythrodermic skin on the back of the hand

Figure 2. Scratch lesions and a reticular pattern seen on the anterior forearm

Screening tests for autoimmune diseases and other diseases such as porphyrias, and serotypes for hepatotropic viruses and HIV were all negative. A skin biopsy (Figs. 3 and 4) showed psoriasiform epidermal hyperplasia with hyperkeratosis alternating with parakeratosis. A slight perivascular lymphocytic infiltrate with verticalization of capillaries in the dermal papillae was seen, compatible with erythroderma and chronic lichenification.

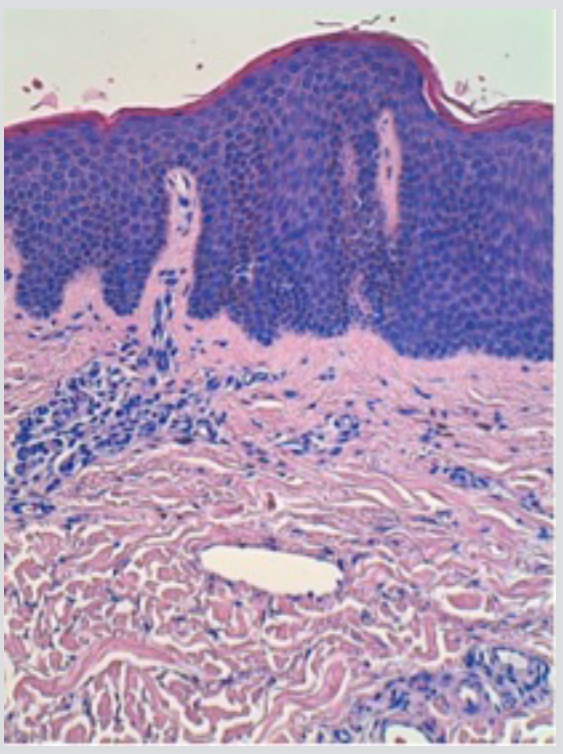

Figure 3. Mild perivascular lymphocytic infiltrate with

verticalization of capillaries in the dermal papillae $(H \& E \times 10)$

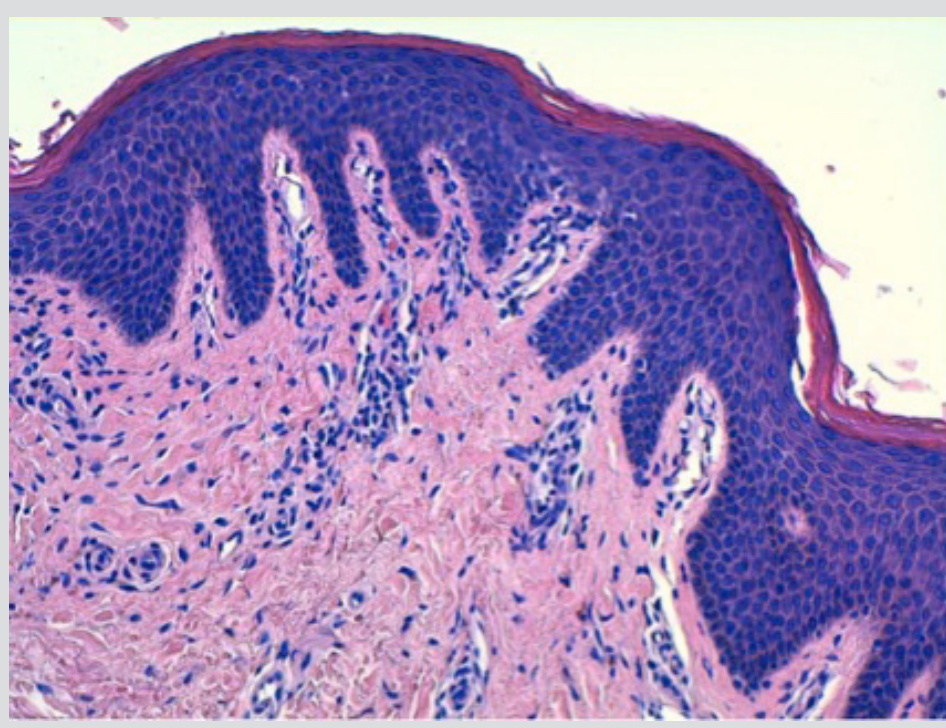

Figure 4. Psoriasiform epidermal hyperplasia with hyperkeratosis alternating with parakeratosis

Since the findings were compatible with erythroderma caused by a drug hypersensitivity reaction, metformin was withdrawn. Prednisone was prescribed at a dose of $1 \mathrm{mg} / \mathrm{kg}$ and treatment with PUVA was initiated.

One month later, the itching had disappeared, the desquamation had decreased and the patient's quality of life had improved. The dosage of corticosteroid was progressively decreased. 


\section{DISCUSSION}

Erythroderma or exfoliative dermatitis is a clinical sign of numerous skin conditions, including drug hypersensitivity in approximately $20 \%$ of cases ${ }^{[1,2]}$.

More than $90 \%$ of the skin is involved and the skin is red with increased local heat with severe skin pain or intense itching ${ }^{[3]}$. Erosions and secondary lichenification can be caused by scratching in chronic erythroderma. In severe forms, the skin is indurated like a 'turtle skin' upon palpation. Desquamation is common, which can be embarrassing for patients in jobs where they interact with the public in person or must wear heavy work clothes in warm conditions.

Erythroderma secondary to metformin is described as a very rare side effect ( 1 in 10,000 treated patients); the condition is more common with penicillins, sulfonamides or allopurinol ${ }^{[4,5]}$. Treatment should include withdrawal of the precipitating drug, and administration of highdose corticoids and PUVA. If there is no improvement after a reasonable time, the case should be re-evaluated with new skin biopsies.

\section{REFERENCES}

1. Li J, Zheng HY. Erythroderma: a clinical and prognostic study. Dermatology 2012;225:154-162.

2. Akhyani M, Ghodsi ZS, Toosi S, Dabbaghian H. Erythroderma: a clinical study of 97 cases. BMC Dermatol 2005;5:5.

3. Rothe MJ, Bernstein ML, Grant-Kels JM. Life-threatening erythroderma: diagnosing and treating the "red man". Clin Dermatol 2005;23:206-217.

4. Ramírez-Bellver JL, Lopez J, Macias E, Fuertes L, Andres I, Alegria V, et al. Metformin-induced generalized fixed drug eruption with cutaneous hemophagocytosis. Am J Dermatopathol 2017;39(6):471-475.

5. Koca R, Altinyazar HC, Yenidünya S, Tekin NS. Psoriasiform drug eruption associated with metformin hydrochloride: a case report. Dermatol Online J 2003 ;9(3):11. 